Frauen besetzen - eine Verdoppelung des Anteils von 15 Prozent im Jahr 2009.

Gesetzliche Quoten sind ein zulässiges und wirksames Instrument zur Beseitigung der Unterrepräsentanz von Frauen in den Spitzengremien der Wirtschaft. Frost und Linnainmaa haben schon 2007 ausgeführt, dass solche Quoten für Aufsichtsräte mit dem Gemeinschaftsrecht und dem Grundgesetz vereinbar wären. ${ }^{17}$ Raasch hat 2009 diese Position vertieft und zudem gezeigt, dass die Quoten in Norwegen inzwischen nicht nur von den Unternehmen im Staatsbesitz, sondern auch von den meisten privaten Unternehmen eingelöst werden konnten und darüber hinaus sogar bei Unternehmen der Frauenanteil in den Führungspositionen gestiegen ist, die gar nicht der Quote unterlagen. ${ }^{18}$ Freiwillig, auch das zeigt das Beispiel Norwegen, sind die Privaten diesen Weg allerdings nicht gegangen. Sie hätten dazu zwei Jahre lang Zeit gehabt, bevor die verbindlichen Quoten in Kraft treten sollten, blieben aber untätig.

Nichts spricht dafür, dass die deutschen Unternehmen im Gegensatz zu diesen norwegischen Erfahrungen auf breiter Front freiwillig gegen ihre gläsernen Decken vorgehen und Frauen an ihre Spitzen bringen werden. Der djb ist daher im Rahmen eines vom BMFSJ geförderten Aktionsprojektes dazu übergegangen, die Vorstände auf Aktionärsversammlungen zu fragen, was sie zur Gleichstellung der Geschlechter und insbesondere zur Verstärkung der Frauenrepräsentanz auf Führungspositionen in ihren Unternehmen im vergangenen Geschäftsjahr getan haben und künftig zu tun gedenken. Der öffentliche Druck auf Unternehmen soll dadurch verstärkt und das Problembewusstsein auf Unternehmens-, aber auch auf Aktionärsseite geschärft werden. Das allein reicht aber keinesfalls aus, um tatsächliche Veränderungen anzustoßen.

Der Staat bleibt weiterhin gefordert, nach EU-Recht und nach dem Gleichberechtigungspostulat des Grundgesetzes. Aber der Koalitionsvertrag „Wachstum. Bildung. Zusammenhalt“ zeigt nicht, dass die Koalitionäre sich dieser Aufgabe ernsthaft stellen wollen, weder bei den Arbeitszeiten noch beim Entgelt und auch nicht zur Verstärkung der Frauenrepräsentanz in Führungspositionen und Aufsichtsräten.

17 Vgl. Frost, Ina Anne/Linnainmaa, Leena, Corporate Governance: Frauen im Aufsichtsrat - Können wir von unseren skandinavischen Nachbarn lernen?, in: Die Aktiengesellschaft 17/2007, $601 \mathrm{ff}$.

18 Vgl. Raasch, Sibylle, Erhöhung der Frauenrepräsentanz in Aufsichtsräten: Norwegen - ein Modell?, in: Zeitschrift für Europarecht, Internationales Privatrecht und Rechtsvergleichung 5/2009, 216 ff.

\title{
Koalitionsvertrag 2009: \\ Bausteine einer modernen, geschlechtergerechten Familien- und Generationenpolitik?'
}

\section{Prof. Dr. Margarete Schuler-Harms}

Vorsitzende der Kommission Recht der sozialen Sicherung, Familienlastenausgleich des djb; Professorin für Öffentliches Recht, insbes. Öffentliches Wirtschafts- und Umweltrecht an der HelmutSchmidt-Universität/Universität der Bundeswehr in Hamburg

\section{Effektivität, Effizienz und Evaluation als Parameter einer „aktivierenden“ Politik}

Das Schiff der Familienpolitik hat im Verlauf der vergangenen drei Legislaturperioden langsam seinen Kurs geändert und zuletzt unter der großen Koalition schnellere Fahrt aufgenommen. Der Koalitionsvertrag schreibt diese Entwicklung fort. Die neue Frauen- und Familienministerin beginnt zusätzliche Akzente zu setzen, scheint den eingeschlagenen Kurs aber grundsätzlich beibehalten zu wollen.

Der Koalitionsvertrag akzentuiert die Aufgabe einer „modernen Familienpolitik für alle Generationen“. Die Steuerund Sozialpolitik stellt er unter den Auftrag größerer Kinderund Familienfreundlichkeit und günstiger Rahmenbedingungen für Familien mit dem Ziel, die Zahl der Geburten zu erhöhen. Die klare Ausrichtung auf Nachwuchssicherung er- fordert - zumal in Zeiten schmaler Kassen - eine zielgenaue Konzeption des Familienleistungsausgleichs. Die Koalitionsparteien versprechen „Wahlfreiheit bei der Gestaltung von Familien- und Erwerbstätigkeit“ und eine Orientierung der Förderung „in der Lebenswirklichkeit von Familien“.

Ein solchermaßen aktivierendes Konzept des Sozial- und Steuerstaates erfordert eine Politik aus einem Guss und damit auch Instrumente, mit denen sich die Wirkung der Maßnahmen auf die Familien beobachten und die Maßnahmen selbst weiterentwickeln lassen. ${ }^{2}$ Unter der Großen Koalition wurde sion Recht der sozialen Sicherung, Familienlastenausgleich. Ergänzend wurden die Informationen des BMFSFJ über „Vorhaben 2010“ für den Ausschuss für Familie, Senioren, Frauen und Jugend des Deutschen Bundestages vom 18.1.2010 herangezogen, abrufbar unter <http://news.einformation.de/v3/client/media/193/ data/20094.pdf> (letztmals überprüft am 12.4.2010). Für weiterführende Hinweise danke ich - stellvertretend - den Kommissionsmitgliedern Prof. Dr. Susanne Dern (Leitung der Fachgruppe Grundsicherung, Arbeitsmarktintegration), Dr. Christine Maurer (Leitung der Fachgruppe Steuerrecht, Kindergeld) und Prof. Dr. Astrid Wallrabenstein (Leitung der Fachgruppe Vorsorge).

2 Zum Konzept des ,aktivierenden Sozialstaats“ näher Kingreen, JZ 2004, 938 (946 f.); Kersten, Die Verwaltung 2007, 309 ff., insbes. 312 f. 
beim Familienministerium ein Kompetenzzentrum eingerichtet und mit der wissenschaftlichen Evaluation der familienbezogenen Transferleistungen begonnen. Auch der Siebte Familienbericht hat verschiedene Handlungsempfehlungen zur Verbesserung, insbesondere zur Wissensvernetzung und zur Evaluation ausgesprochen. ${ }^{3}$ Die Koalition kündigt nun an, diese Evaluation fortzusetzen und entsprechende Instrumente hierzu zu entwickeln. Leistungen sollen - im Lichte der oben beschriebenen Zielsetzungen - wirksamer und effizienter gestaltet und gebündelt werden, Unterhalts-, Steuer- und Sozialrecht im Lichte dieser Zielsetzungen stärkere Harmonisierung erfahren. ${ }^{4}$

Ähnliche Ansätze gibt es für die Gleichstellungspolitik. Der Erste Bundesgleichstellungsbericht soll noch in diesem Jahr fertig werden. Auf Initiative der Bundesregierung nimmt dieser die Lebensverlaufsperspektive auf. ${ }^{5}$ Gleiches gilt für den „Rahmenplan zur gleichberechtigten Teilhabe von Frauen und Männern“, den die Koalitionspartner vereinbart haben.

\section{Nachwuchssicherungspolitik durch Förderung von Repro- duktion, Bildung und Integration}

Der Koalitionsvertrag kündigt an, jedem neugeborenen Kind „beispielsweise ein Zukunftskonto mit einem Startguthaben von 150 Euro“ einzurichten und „Einzahlungen bis zur Volljährigkeit mit einer Prämie zu unterstützen“. Eine Zweckbindung, auf die die Positionierung der Vereinbarung unter dem Titel „Bildungsfinanzierung“ schließen lassen könnte, ist nicht vorgesehen. Kindergeld und Kinderfreibeträge wurden bereits zum 1. Januar 2010 erhöht, ${ }^{6}$ freilich mit zweifelhaften ökonomischen Effekten. Das zusätzliche Geld schöpfen die Not leidenden Kommunen bei einigen Familien nämlich schon jetzt über höhere Gebühren und Beiträge ab. Ob die Koalition damit wirksame „Familienförderung“ betrieben hat, sollte sie sorgfältig evaluieren.

Die nahezu einhellige Forderung des Schrifttums nach Entkoppelung von Einkommensteuer- und Kindergeldrecht ${ }^{7}$ haben die Koalitionspartner nicht aufgenommen. Sie wollen stattdessen prüfen, ob die von den Familienkassen durchgeführte Auszahlung des Kindergeldes einer anderen Stelle übertragen werden kann. Weiterhin wollen sie prüfen, ob weitere steuerfinanzierte familienpolitische Leistungen zusammengefasst werden können. Ob hierbei an eine „Kindergrundsicherung “ gedacht wird, ${ }^{8}$ lässt der Koalitionsvertrag nicht erkennen.

Vorschulische Kinderbetreuung ist für die Koalition kein generelles Bildungsthema, offenbar aber eines der „Integration durch Bildung“. Unter der Zielsetzung, die Integrationskraft von Kindergärten und Schulen zu verstärken, sprechen sich die Koalitionspartner für einen bedarfsgerechten Ausbau der frühkindlichen Bildungseinrichtungen und der Ganztagsschule aus. Generell sollen die Betreuungsrelationen von Schulen und Kindertagesstätten verbessert und in die Qualifikation der Erzieherinnen und Erzieher investiert werden. Diese Passagen des Koalitionsvertrags stehen freilich unter dem Vorbehalt einer großen Bereitschaft der Länder zur akti- vierenden frühkindlichen Bildungspolitik und zum Zusammenwirken mit dem seinerseits zu finanziellen Investitionen bereiten Bund.

\section{Stärkung der Freiheit, Familienleben und Erwerbstätigkeit nach eigenen Wünschen zu gestalten}

Für die Verbesserung der demografischen Bilanz setzen die Koalitionspartner auf „Wahlfreiheit“ von Frauen, Eltern und Familien. Dieser Begriff ist äußerst dehnbar, denn er umfasst jedenfalls begrifflich sowohl die Förderung der Nichterwerbstätigkeit von erziehenden Eltern (respektive Müttern) und pflegenden Angehörigen als auch die Stärkung der Vereinbarkeit von Familienleben und Erwerbstätigkeit. ${ }^{9}$ Entsprechend offen und indifferent sind die unter diesem Dach vereinten politischen Konzepte, an denen die Koalitionspartner auch nicht grundsätzlich rütteln.

Einerseits bleibt bei der Förderung der Nichterwerbstätigkeit nicht nur vieles beim Alten, es wird sogar Neues in Aussicht genommen. Das Ehegattensplitting mit seinen beschäftigungsfeindlichen Effektiven ${ }^{10}$ wird nicht angetastet. An der Figur der geringfügigen Beschäftigung soll nicht nur festgehalten, sondern diese sozial- wie frauenpolitisch kontraproduktive Beschäftigungsform weiter ausgebaut und sogar „dynamisiert“ werden. „Minijobs“ sind für Männer zumeist Nebenjobs neben der Haupterwerbstätigkeit. Für Frauen bilden sie vielfach die einzige und oft eine sehr prekäre Erwerbsquelle. Gefördert wird ein Vollzeit-/Teilzeitmodell, das den Wünschen und Interessen von Frauen und Familien nur in begrenztem Maße Rechnung trägt. „Normalarbeitsverhältnisse“ und „kontinuierliche Erwerbsbiografien“, denen ein Thema des

3 „Familie zwischen Flexibilität und Verlässlichkeit - Perspektiven für eine lebensverlaufsbezogene Familienpolitik, BT-Drs. 16/1360 vom 26.4.2006, $292 \mathrm{ff}$.

4 Vgl. a. Scheiwe, djbZ 2009, $123 \mathrm{ff}$.

5 Vgl. BMFSFJ vom 23.6.2009, abrufbar unter <http://www.bmfsfj. de>, Stichwort: Gleichstellungsbericht (Zugriff 20.4.2010).

$6 \S 32$ Abs. $6, \S 66$ Abs. 1 S. 1 EStG i.d.F. des Wachstumsbeschleunigungsgesetzes vom 22.12.2009, BGBI. I, 3950 ff.

7 Z.B. Felix, Die Familie zwischen Privatrecht, Sozialrecht und Steuerrecht, in: Mellinghoff (Hrsg.), Steuern im Sozialstaat, 2006, 150 ff.; Axer, a.a.O., 175 ff.; Jachmann, BB 2008, $591 \mathrm{ff}$.

8 Entsprechende Forderungen und Vorschläge z.B. bei Bündnis Kindergrundsicherung „Kinder brauchen mehr!“ mit der Forderung einer Grundsicherung von 502 Euro pro Kind; zu Konzept und Bündnispartnern näher unter 〈http://www.kinderarmut-hat-folgen.de> (Zugriff 20.4.2010); Lenze, Die Verfassungsmäßigkeit eines einheitlichen und der Besteuerung unterworfenen Kindergeldes, HansBöckler-Stiftung, Arbeitspapier 151, 2008; I. Becker, WSI Mitt. 2008, 139 ff.; Hauser/Becker, Vom Kinderzuschlag zum Kindergeldzuschlag - ein Reformvorschlag zur Bekämpfung von Kinderarmut, SOEPpapers on Multidisciplinary Panel Data Research at DIW Berlin, Nr. 87, Berlin 2008.

9 Vgl. dazu auch den Beitrag von Klug/Kocher/Raasch in diesem Heft.

10 Zu Rechtsentwicklung und Aktivitäten des djb im Steuerrecht Deutscher Juristinnenbund e.V. (Hrsg.), Juristinnen in Deutschland. Die Zeit von 1900 bis 2003, 4. Aufl. 2003, 150 ff.; zum Ehegattensplitting Diwell/Schuler-Harms, Plädoyer zur Abschaffung des Ehegattensplitting, in: Müller-Magdeburg (Hrsg.), Festschrift für Lore Maria Peschel-Gutzeit, 2002, 44 ff. m.w.Nw.; Hintergrundpapier vom 27.6.2006 m.w.Nw. (abrufbar unter 〈http://www.djb.de> unter „Stellungnahmen“); Maurer/Spangenberg, djbZ 2009, $102 \mathrm{f}$. 
diesjährigen Deutschen Juristentags gewidmet ist, ${ }^{11}$ fördern solche Rahmenbedingungen gerade nicht.

Zum umstrittenen Betreuungsgeld formuliert der Koalitionsvertrag: „Um Wahlfreiheit zu anderen öffentlichen Angeboten und Leistungen zu ermöglichen, soll ab dem Jahr 2013 ein Betreuungsgeld in Höhe von 150,- Euro, gegebenenfalls als Gutschein, für Kinder unter drei Jahren als Bundesleistung eingeführt werden. “ Der djb hat sich wie viele andere Verbände $^{12}$ gegen ein Betreuungsgeld ausgesprochen. ${ }^{13}$

Auf der anderen Seite werden viele Anstrengungen angekündigt, die Vereinbarkeit von Familienleben und Erwerbstätigkeit zu fördern. Der Koalitionsvertrag verspricht Bemühungen um ein qualitativ und quantitativ hochwertiges Angebot an außerhäuslicher Kinderbetreuung und verschreibt sich der Qualitätssicherung und Verbesserungen bei der Ausbildung in den Erziehungsberufen und der Tagespflege. Zum prekären Übergang vom Elterngeldbezug in die Erwerbstätigkeit wegen fehlender Kinderbetreuungseinrichtungen enthält der Koalitionsvertrag leider nichts.

Das Elterngeld kann schon kurze Zeit nach seiner Einführung als gesellschaftlich und politisch akzeptiert gelten. Die Koalitionspartner versprechen eine Überarbeitung zu vier Punkten, die - wie das Elterngeld insgesamt - keine klare Zielorientierung erkennen lässt. Erstens soll das Elterngeld flexibilisiert, nämlich als Teilelterngeld über einen längeren Zeitraum (28 Monate) gestreckt werden können. Zweitens soll gleichzeitige Elternzeit beider Eltern nicht mehr zu doppeltem Anspruchsverbrauch führen - eine wichtige Neuerung zur Absicherung partnerschaftlicher Erziehungsmodelle. Drittens sollen die Partnerschaftsmonate „gestärkt“ werden; eine Ausweitung der sogenannten „Vätermonate“ von zwei auf vier steht im Raum. Und viertens wird die komplizierte Einkommensermittlung bei Selbständigen überarbeitet. Ein Referentenentwurf zum Elterngeld wird noch in der ersten Jahreshälfte erwartet.

Der demografische Wandel stellt besondere Anforderungen an die Organisation der Pflege. Die politischen Absichtserklärungen und Programme verdienen große Aufmerksamkeit des djb, nicht zuletzt weil sowohl in der Angehörigenpflege als auch in der professionellen Pflege Frauen überwiegen. Durch besondere Maßnahmen wollen die Koalitionspartner „Familien ... eine Chance geben, Erwerbstätigkeit und die Unterstützung der pflegebedürftigen Angehörigen besser in Einklang zu bringen“. Ein lohnkostenneutraler Anspruch auf Pflegezeit, wie von der neuen Familienministerin vorgeschlagen, muss auch im djb sorgfältig und konstruktiv diskutiert werden. Die Absicherung von nicht erwerbsmäßigen Pflegezeiten in der Alterssicherung ist noch nicht befriedigend gelöst.

Die Koalitionsparteien wollen außerdem „dafür sorgen, dass ausländische Hilfskräfte ebenso wie pflegende Angehörige oder deutsche Hilfskräfte auch notwendige pflegerische Alltagshilfen erbringen können“. Mehr als ein allgemeiner Merkposten ist dies nicht. Dringend erforderlich wären angemessene zuwanderungs-, arbeits- und sozialrechtliche Rahmenbedingungen für im Privathaushalt beschäftigte Hilfskräf- te. Konkreter werden die Koalitionspartner für den Bereich der berufsmäßigen Altenpflege. Das Berufsbild soll attraktiver gestaltet, die Pflegeberufe sollen in der Ausbildung durch ein neues Berufsgesetz grundlegend modernisiert und zusammengeführt werden.

\section{Grundsicherung und Arbeitsförderung}

Die Reform der Grundsicherung wird einen Schwerpunkt der sozialpolitischen Gesetzgebung in 2010 bilden. Die im Koalitionsvertrag angekündigten Reformvorhaben - SGB II-Strukturreform, Erhöhung des Freibetrags beim Schonvermögen und Verbesserung der Hinzuverdienstregelungen - sind nach der Entscheidung des BVerfG vom 9. Februar $2010^{14}$ um weitere zu ergänzen. Zusätzlich sind nun die Kinderregelsätze erstmals und die Regelsätze für Erwachsene neu zu bemessen; auch die Berücksichtigung außergewöhnlicher Belastungen soll in einer Härtefallregelung anders als bisher zu berücksichtigen sein. ${ }^{15}$

Die SGB II-Reform wird auch den djb in diesem Jahr beschäftigen - allerdings mit anderen als den im Koalitionsvertrag genannten Schwerpunkten. In einem vom BMFSFJ geförderten Forschungsprojekt haben Prof. Dr. Ursula Rust ${ }^{16}$ und Prof. Dr. Sigrid Betzelt für den djb aus rechts- und sozialwissenschaftlicher Sicht die Individualisierung von Leistungen des SGB II unter Berücksichtigung von Unterhaltspflichten untersucht. ${ }^{17}$ Ein Nachfolgeprojekt widmet sich den gleichstellungspolitischen Anforderungen im laufenden SGB II-Reformprozess. Gleichstellungspolitische Desiderate hat der djb bereits in einer Presseerklärung anlässlich der BVerfG-Entscheidung vom 9. Februar formuliert. ${ }^{18}$

Reformbedürftig ist die Methode der Bedarfsberechnung. Derzeit gilt die sogenannte horizontale Berechnungsmethode, nach der das individuelle Einkommen einer Person allen an-

11 68. DJT 21.-24.9.2010 in Berlin, Sektion Arbeits- und Sozialrecht: „Abschied vom Normalarbeitsverhältnis?" Näheres unter <http:// www.djt.de> (Zugriff 20.4.2010).

12 Insbes.,Betreuungsgeld ist ein sozial- und gleichstellungspolitischer Rückschritt“, Offener Brief vom 2. Dezember 2009, z.B. abrufbar unter <www.vamv.de/uploads/media/Offener_Brief_Betreuungsgeld.pdf> (Zugriff 19.4.2010); Stellungnahme des Deutschen Kinderschutzbundes Bundesverband e.V. zum geplanten Betreuungsgeld vom 25.11.2009, abrufbar unter <http://www.dksb.de/ images/web/og-11-25_Stellungnahme\%2oBetreuungsgeld.pdf> (Zugriff 19.4.2010).

13 Pressemitteilung des djb vom 28.2.2008 und Stellungnahme zum Entwurf eines Gesetzes zur Förderung von Kindern unter drei Jahren in Tageseinrichtungen und in der Kindertagespflege (Kinderförderungsgesetz - KiFöG) vom 4.4.2008.

$141 \mathrm{BvL}$ 1/o9, 1 BvL 3/og, 1 BvL 4/o9.

15 Vgl. dazu die Geschäftsanweisung des BMAS vom 17.2.2010 - GeschZ SP II-II-1303 / 7000/5215, abrufbar unter <http://www.arbeitsagentur.de>, Stichwort „Geschäftsanweisung Härtefallregelung“ (Zugriff 17.2.2010).

16 Mitglied im djb und Vorsitzende der Kommission Familienlastenausgleich von 1999 bis 2001.

17 Betzelt/Rust, Individualisierung von Leistungen des SGB II unter Berücksichtigung der familialen Unterhaltsverpflichtungen, erscheint vorauss. 2010; vgl. a. Rust, Entlastung des Staates oder Entlastung der Familie?, in: Knickrehm/Rust (Hrsg.), Arbeitsmarktpolitik in der Krise. Festgabe für Karl-Jürgen Bieback, 2010, S. $141 \mathrm{ff}$.

18 Pressemitteilung vom 9.2.2010; vgl. a. Rust, djbZ 2009, 116 ff. 
deren mit ihr in einer „Bedarfsgemeinschaft“ lebenden Personen zugerechnet wird. Diese Berechnungsmethode führt neben vielen anderen Problemen ${ }^{19}$ - dazu, dass - anders als im Sozialhilferecht - auch solche Personen als hilfebedürftig angesehen werden, die genug Einkommen erzielen, um sich selbst zu unterhalten. Für diese Personen wird die Hilfebedürftigkeit also „fingiert“. Verbunden wird das mit der Pflicht, den „eigenen“ Lebensunterhalt so zu sichern, dass die - normativ begründete - Bedürftigkeit beseitigt wird. ${ }^{20}$ Teilzeitbeschäftigte Frauen etwa können zur Aufgabe der Teilzeittätigkeit und zur Aufnahme einer Vollzeitbeschäftigung aufgefordert werden.

Erhebliche Probleme wirft auch die Regelung des $\mathbb{9}$ Abs. 2 S. 2 SGB II auf, nach der das Einkommen auf Kinder des Partners oder der Partnerin anzurechnen ist, zu denen kein zivilrechtliches Unterhaltsverhältnis besteht (sog. faktische Stiefkinder). Die verfassungsrechtlichen Bedenken sind durch das Urteil des BSG vom 13. November 2008 nicht ausgeräumt. ${ }^{21}$ Kindern wird durch diese Berechnung der Anspruch auf Grundsicherung gemindert oder gar versagt ohne Rücksicht darauf, ob die faktischen „Stiefeltern“ zu zusätzlichen Leistungen für die Lebensunterhaltssicherung an das Kind überhaupt bereit sind. Für erwachsene Kinder unter 25 Jahren ist diese Regelung besonders problematisch, denn ihnen wird zugleich im Falle der Hilfebedürftigkeit die Begründung eines eigenen Hausstandes erheblich erschwert $(\mathbb{S} 22$ Abs. 2a SGB II). Der Gesetzgeber hat die sogenannte Stiefkind-Regelung damit begründet, dass eine Benachteiligung von Ehen gegenüber nichtehelichen Lebensgemeinschaften beseitigt werden solle. ${ }^{22}$ Tatsächlich dürfte gerade diese Regelung viele Alleinstehende mit Kindern daran hindern, mit dem neuen Partner zusammenzuziehen.

Für die Anrechnung des Kindergeldes als Einkommen des Kindes wird wenig sachgerecht differenziert zwischen Kindern, die zur Bedarfsgemeinschaft gehören, und solchen, die außerhalb des Haushalts leben. ${ }^{23}$ Die Regelung des $\mathbb{} 11$ Abs. 1 S. 3 SGB II für in der Bedarfsgemeinschaft lebende Kinder widerspricht außerdem der Wertung im neu gefassten $\ 1612$ b BGB, wonach das Kindergeld im Regelfall den Barbedarf des Kindes mindern soll.

Nicht angemessen werden schließlich Personen behandelt, die bei individueller Betrachtung leistungsberechtigt wären, wegen Hinzurechnung fremden Einkommens oder Bezugs von Kinderzuschlag nach $\$ 6$ a $\mathrm{BKGG}^{24}$ aber die Leistungsberechtigung verlieren (sog. Nichtleistungsbezieher/innen). Die Arbeitsagenturen fördern vorrangig und intensiv arbeitslos gemeldete Leistungsberechtigte. Ihre Vermittlung in den Arbeitsmarkt führt nicht nur zu Einsparungen, sondern bessert auch die Arbeitsmarktstatistik auf, in der Nichtleistungsbezieher/innen gar nicht geführt werden. Die Effekte sind geschlechtsspezifisch, denn häufig schließt das höhere Einkommen des männlichen Partners den Anspruch der Frau auf Leistungen nach SGB III aus. Arbeitsmarktpolitische Verbesserungen für diese Personengruppe sieht der Koalitionsvertrag nicht vor.

\section{Ein Maßnahmenpaket für Alleinerziehende}

Kinder allein aufzuziehen stellt Eltern vor besondere Herausforderungen und bildet zugleich ein erhebliches Armutsrisiko. Diese Herausforderungen und Risiken treffen ganz überwiegend Frauen. Trotz langjähriger Bemühungen um eine verbesserte Familienpolitik wächst die Gruppe alleinerziehender Mütter mit minderjährigen Kindern in Armut. ${ }^{25}$ Die Fehlförderung ist für diese Gruppe von Familien evident, die im Koalitionsvertrag angekündigte Verbesserung der Rahmenbedingungen deshalb durch Art. 6 Abs. 1 GG geradezu geboten. ${ }^{26}$

In das angekündigte „Maßnahmenpaket“ könnte nach Vorstellung der Koalitionspartner eine Umgestaltung des steuerlichen Entlastungsbetrags ( $\mathbb{S} 24$ a EStG) etwa zum Abzug von der Steuerschuld einfließen. Ob der Entlastungsbetrag die geminderte Leistungsfähigkeit der Alleinerziehenden berücksichtigt oder dazu dient, das Armutsrisiko abzufedern, hat das Bundesverfassungsgericht in einer Kammerentscheidung von Juni 2009 offengelassen. ${ }^{27}$ Entsprechend groß ist der Gestaltungsspielraum des Gesetzgebers.

In das Maßnahmenpaket zählt auch die Flexibilisierung und „Entbürokratisierung“ des Unterhaltsvorschusses und seine Ausweitung bis zur Vollendung des vierzehnten Lebensjahrs.

Die schlecht koordinierten Übergänge nach dem Bezug des Elterngeldes und vor dem regulären Anspruch auf einen Kinderbetreuungsplatz ab dem vierten Lebensjahr treffen alleinerziehende Eltern besonders schwer und führen sie besonders häufig in niedrige Einkommen, den Bezug von Kinderzuschlag nach $\mathbb{6}$ 6a BKGG oder von Leistungen nach SGB II. Im Rahmen des Maßnahmenpakets bedürfen deshalb auch die Wechselwirkungen von Kinderbetreuungsangebot, Grundsicherungsleistung und Arbeitsmarktintegration eingehender Überprüfung. Erwerbsfähigen Eltern ist eine Arbeit nicht zumutbar, wenn sie die Erziehung eines Kindes gefährden würde. $\$ 10$ Abs. 1 Nr. 3 SGB II stellt hierzu die Vermutung auf, dass die Erziehung eines in der Tageseinrichtung oder in Tagespflege betreuten Kindes nicht gefährdet ist - allerdings erst für Kinder ab dem vierten Lebensjahr. Im Zusammenwirken von

19 Ausführlich und instruktiv Länder-Arbeitsgruppe „Maßnahmen zur Verminderung der Belastung und zur Effizienzsteigerung der Sozialgerichte“. Empfehlungen vom 19.10.2009, abrufbar unter <http:// www.berlin.de/imperia/md/content/senatsverwaltungen/justiz/ aktuell/empfehlungen_der_arbeitsgruppe.pdf> (Zugriff 19.4.2010), $71 \mathrm{ff}$.

20 Länder-Arbeitsgruppe (Fn. 19), Empfehlungen vom 19.10.2009, 71.

21 BSG vom 13.11.2008 B 14 AS 2/o8 R. Weiterhin kritisch z.B. Länder-Arbeitsgruppe, (Fn. 19), 75 f.; eine Verfassungsbeschwerde ist anhängig (Az. 1 BvR 1083/o9).

22 BT-Drs. 16/1410, 20.

23 Zu Einzelheiten vgl. Länder-Arbeitsgruppe, (Fn. 19), 85 f.

24 Hierzu Dern, djbZ 2009, 120 ff. m.w.Nw.

25 Vgl. nur Dritter Armuts- und Reichtumsbericht der Bundesregierung, 2008, $87 \mathrm{ff}$., abrufbar unter <http://www.bmas.bund.de> (Zugriff 20.4.2010); BA, Arbeitsmarktberichterstattung: Alleinerziehende im SGB II, 2008, 5 ff.

26 Die verfassungsrechtliche Relevanz evidenter Fehlförderung betont BVerfGE 87, 1 (39).

27 - 2 BvR 310/07 - zu $\$ 24 b$ EStG (Entlastungsbetrag für Alleinerziehende), abrufbar unter 〈http://www.bverfg.de/entscheidungen〉, 12 f. (Zugriff 20.4.2010). 
Kommunen, Bundesagentur für Arbeit und Jobcentern und selbst in der Öffentlichkeit scheint sich die Meinung zu verbreiten, dass Eltern mit Kindern unter drei Jahren weder Leistungen der Arbeitsmarktförderung beanspruchen können noch ihnen - mangels Arbeitsplatz - ein Kinderbetreuungsplatz vorrangig zuzuweisen ist. Hier bedarf es zumindest einer besseren, den Willen des Gesetzes und die Arbeitsbereitschaft alleinerziehender Eltern aufnehmenden Umsetzung.

\section{Alterssicherung}

Unter dem Titel „Verbesserung der Kindererziehung in der Alterssicherung “ enthält der Koalitionsvertrag nur die Erklärung, im Rahmen der finanziellen Möglichkeiten zu prüfen, „wie wir die familienpolitische Komponente stärken und deshalb Erziehungsleistungen in der Alterssicherung noch besser berücksichtigen können“. Das klingt angesichts leerer Kassen und einer nicht unprekären Lage der Rentenversicherung wenig ermutigend. Der djb wird also weiterhin sehr aktiv ein Rentensystem einfordern müssen, in dem das Aufziehen von Kindern als „Nachhaltigkeitsfaktor“ angemessene Berücksichtigung findet und in dem Nutzen und Lasten zwischen Frauen und Männern gerechter verteilt werden. Zu den Mindestforderungen gehört seit Langem die Aufstockung der Kindererziehungszeiten von ein auf drei Jahre auch für Geburten vor 1992. ${ }^{28}$ Es ist nicht gerecht, gerade den Jahrgängen nur ein Erziehungsjahr gutzuschreiben, die während der Erziehungsphase ohnehin geringere staatliche Leistungen erhalten haben als die jüngeren Familien.

Da sich die Koalitionspartner zur staatlich geförderten Altersvorsorge „,bekennen“, ist daran zu erinnern, dass auch kapitalgedeckte Vorsorgesysteme demografieanfällig sind und auch für sie das Aufziehen von Kindern einen „Nachhaltigkeitsfaktor" bildet. ${ }^{29}$ Die sogenannte Riester-Förderung bildet die Kindererziehungskomponente ab. Auch für die berufsständischen Versorgungswerke wäre eine obligatorische Berücksichtigung der Kindererziehung, wie sie einige Versorgungswerke ansatzweise kennen, systemgerecht. Die Berücksichtigung von Kindererziehungszeiten für Anwältinnen, Ärztinnen und Architektinnen in der gesetzlichen Rentenversicherung ist nur die zweitbeste Lösung. Die Betroffenen werden an zwei Rentenversicherungsträger verwiesen, was größeren Aufwand und Unsicherheiten mit sich bringt. Außerdem werden für die Kindererziehung nicht die versorgungswerkspezifischen Durchschnittseinkommen, sondern das Durchschnittseinkommen der gesamten Bevölkerung angerechnet. Im System bleiben die Kindererziehungsleistungen als „Nachhaltigkeitsfaktor" berufsständischer Systeme ausgeblendet.

Alterssicherung erfolgt auch durch leistungsgerechte Vorsorgesysteme für den Fall der Pflegebedürftigkeit. Die Koalitionspartner vereinbaren hierzu erstens den Willen zu einer neuen, differenzierteren Definition der Pflegebedürftigkeit, zweitens (s.o. „Stärkung der Freiheit, Familienleben und Erwerbstätigkeit nach eigenen Wünschen zu gestalten“) die Optimierung sowohl der nicht erwerbsmäßigen als auch der erwerbsmäßigen Pflege und drittens die Ergänzung des beste- henden Umlageverfahrens durch eine verpflichtende, kapitalgedeckte Versicherung.

Eine kapitalgedeckte - und daher voraussichtlich private Zusatzpflegeversicherung soll nach Vorstellung der Koalitionspartner verpflichtend, individualisiert und generationengerecht ausgestaltet sein. Die Forderung der Generationengerechtigkeit wird nicht näher präzisiert, ${ }^{30}$ ist aber hoffentlich so zu verstehen, dass die staatliche Förderung ähnlich wie beim „Riester“ nach Kinderzahl gestaffelt werden soll. Eindeutig ist das nicht, denn Beiträge in der gesetzlichen Pflegeversicherung werden nur danach differenziert, ob die Versicherungsnehmer „Eltern“ sind, d.h. irgendwann einmal Kinder aufgezogen haben $(\mathbb{S} 55$ Abs. 3 S. 2 SGB XI i.V.m. $\mathbb{S} 56$ Abs. 1 Nr. 3, Abs. 3 Nr. 2 und 3 SGB I), oder nicht. ${ }^{31}$ Diese Gestaltung berücksichtigt nicht die individuelle Leistungsfähigkeit der erziehenden Eltern, sondern „honoriert“ Kindererziehung wenig differenziert und generationengerecht. Als Vorbild für eine staatliche Förderung privater Pflegevorsorge eignet sie sich deshalb nicht.

Eine geschlechtergerechte Tarifgestaltung der privaten Pflegevorsorge spricht der Koalitionsvertrag nicht an. Sie ist jedoch europarechtlich gefordert ${ }^{32}$ und sollte nach der Rechtsentwicklung zur ergänzenden privaten Altersvorsorge ${ }^{33}$ in staatlicher Gewährleistungsverantwortung selbstverständlich sein.

28 Stellungnahme des djb vom 7.12.2000 „Thesen zur Rentenstrukturreform“, unter II.3. Zu den weiteren Forderungen und Vorschlägen aus dem djb vgl. die Beiträge von Heinze, Riedel und Rust, djbZ 2009, 108 f., $109 \mathrm{ff}$., $114 \mathrm{ff}$.

29 Aus der ökonomischen Literatur statt vieler Färber, Generationengerechtigkeit, in: LVA Rheinland-Pfalz, Zukunft der Rente - Rente der Zukunft, 2004, 17 (25); Schmähl/Rothgang/Viebrock, Berücksichtigung von Familienleistungen in der Alterssicherung, 2006, $52 \mathrm{ff}$.

30 Dazu ausführlich und mit weiteren Nachweisen z.B. Baer, VVDStRL 68 (2008), 290 ff.; Kluth, VVDStRL 68 (2008), 246 ff.; Schuler-Harms, DVBI. 2008, $1090 \mathrm{ff}$.

31 Eingeführt durch das Kinderberücksichtigungsgesetz vom 15.12.2004, BGBI. I, 3448; dagegen Vorschlag eines pauschalen Abzugsbetrags vom einkommensproportionalen Versicherungsbeitrag pro Kind in der Stellungnahme des djb vom 10.6.2004.

32 Art. 5 RL 2004/113/EG vom 13.12.2004, ABI. L 373/37.

33 Zur Diskussion um Unisex-Tarife für die private Altersvorsorge z.B. Temming, ZESAR 2005, $72 \mathrm{ff}$. Vgl. a. Stellungnahme des djb vom 4.4.2004 für private Kranken- und Rentenversicherungen. 